\title{
Nonlinear Predictive Control for Durability Enhancement and Efficiency Improvement in a Fuel Cell Power System
}

\author{
Julio Luna ${ }^{\mathrm{a}, *}$, Samir Jemei ${ }^{\mathrm{b}, \mathrm{c}}$, Nadia Yousfi-Steiner ${ }^{\mathrm{b}, \mathrm{c}, \mathrm{d}}$, Attila Husar ${ }^{\mathrm{a}}$, Maria Serra ${ }^{\mathrm{a}}$, Daniel \\ Hissel $^{\mathrm{b}, \mathrm{c}}$ \\ ${ }^{a}$ Institut de Robòtica i Informàtica Industrial (CSIC-UPC). C/Llorens i Artigas 4-6. 08028 Barcelona, Spain \\ ${ }^{b}$ FEMTO-ST UMR CNRS 6174, University of Bourgogne Franche-Comté (UBFC), France \\ ${ }^{c}$ FCLAB (FR CNRS 3539), Belfort, France \\ ${ }^{d} L A B E X$ ACTION CNRS, France
}

\begin{abstract}
In this work, a nonlinear model predictive control (NMPC) strategy is proposed to improve the efficiency and enhance the durability of a proton exchange membrane fuel cell (PEMFC) power system. The PEMFC controller is based on a distributed parameters model that describes the nonlinear dynamics of the system, considering spatial variations along the gas channels. Parasitic power from different system auxiliaries is considered, including the main parasitic losses which are those of the compressor. A nonlinear observer is implemented, based on the discretised model of the PEMFC, to estimate the internal states. This information is included in the cost function of the controller to enhance the durability of the system by means of avoiding local starvation and inappropriate water vapour concentrations. Simulation results are presented to show the performance of the proposed controller over a given case study in an automotive application (New European Driving Cycle). With the aim of representing the most relevant phenomena that affects the PEMFC voltage, the simulation model includes a two-phase water model and the effects of liquid water on the catalyst active area. The control model is a simplified version that does not consider two-phase water dynamics.
\end{abstract}

Keywords: proton exchange membrane fuel cell, distributed parameter system, nonlinear observer, model predictive control, degradation, starvation

\section{Introduction}

Governments, industry and society in general are becoming aware of the problems derived from the energy dependency on fossil fuels and other non-renewable energies. In this context, proton exchange membrane fuel cells (PEMFC), which have hydrogen as fuel, are gaining increasing attention as clean and efficient energy conversion devices for a broad range of applications, such as automotive, stationary, combined heat and power (CHP) and portable systems.

To operate properly, different physical variables have to be measured from the PEMFC. This makes it possible to implement feedback control techniques that can improve the lifetime and

*Corresponding author. Tel: +349340158 05 — Fax: +34934015750.

Email address: jluna@iri.upc.edu (Julio Luna) 
efficiency of the system. Specifically, efficiency and degradation of the PEMFC are greatly affected by its internal conditions. However, while some of the internal variables can be measured using the existing sensor technology, there are parts of the system that are inaccessible. Henceforth, dynamic fuel cell modelling $[1,2,3,4]$, fuel cell model-based control $[5,6,7]$ and model-based observation and identification of parameters $[8,9,10,11]$ are compelling research topics in the field.

Regarding the efficiency in PEMFC-based systems, besides the hydrogen supply subsystem, the air supply subsystem plays a crucial role [12]. In particular, the air pressure at the cathode and the oxygen excess ratio are directly related with the efficiency of the system and thus, this subsystem has to be considered in a control strategy aiming at efficiency improvement $[13,14,15]$.

Concerning degradation, the lifetime of PEMFCs is mainly reduced as a result of catalyst metal degradation and carbon-support corrosion. Both of these degradation mechanisms are linked and supplement each other $[16,17,18]$ because the platinum $(\mathrm{Pt})$ catalyses the carbon-support oxidation. At the same time, the loss of carbon releases carbon-supported Pt particles and therefore it produces active surface area loss. In the literature, accelerated durability tests have been carried out to study degradation mechanisms in PEMFCs [19] and other types of fuel cells [20].

Three degradation categories can be distinguished [21]: baseline degradation, cycling degradation and incident-induced degradation. The baseline degradation is due to long-term material degradation and it is unavoidable (it exists as long as the fuel cell is operating). Moreover, degradation is accelerated by cycling conditions [21]. Finally, severe degradation occurs when the fuel cell is subject to an unexpected incident which may cause global or local reactant starvation. Controllers can aid to avoid starvation-induced degradation and thus reduce the impact of cycling as well as the impact of unexpected operating changes.

Using advanced control techniques that consider the inherent nonlinear behaviour of PEMFC systems, the improvement of efficiency and durability can be achieved. Model predictive control (MPC) has an intrinsic capability of considering several manipulable variables and control objectives (multi-objective control) as well as the capability to deal with systems constraints in a systematic and straightforward manner [22]. These properties make MPC a promising control strategy for PEMFC-based systems.

A common situation in PEMFC-based energy systems is that the fuel cell works in a wide range of dynamics and power demands. The nonlinear MPC (NMPC) approach [23, 24] takes into consideration the proper system dynamics in the whole range of operation and integrates them into a closed-loop control scheme. Nonetheless, one of the main problems that can be encountered when using this control strategy is the high computational burden.

Until now, most of the works focused on PEMFC control have addressed the improvement of PEMFC efficiency [25] and durability [26] separately. The present paper proposes a global solution to tackle the efficiency and durability improvement of the PEMFC power system at the same time, using state-of-the-art nonlinear control and observation techniques. The simulation results consider the New European Driving Cycle (NEDC) as the case study.

The main contribution of this paper relies on the development of a NMPC strategy based on a nonlinear distributed parameters model [3] of a PEMFC power system. Due to implementation reasons, the control model is a simplified version of the simulation model and it does not have the two-phase water model. The controller objective is to maximise the efficiency of the system and to limit the internal gas concentration distributions in order to improve the durability of the PEMFC avoiding possible local starvation scenarios. The control strategy makes use of a nonlinear distributed parameters observer (NDPO) [9] to estimate the fuel cell internal conditions which are restricted in the optimisation problem. The PEMFC simulation model used in this 
work is a distributed parameters model derived from the discretisation of the partial differential equations that describe the nonlinear dynamics of the system, considering spatial variations along the gas channels. In addition, for a more realistic description of the PEMFC voltage dynamics, the cathode side of the PEMFC includes a two-phase multi-scale water transport model that combines macroscopic two-phase flow of water with mesoscopic pore filling effects in the diffusion and catalyst layers [27]. Moreover, the cathode is fed with a compressor whose modelled parasitic demand is considered in the control objective of net efficiency improvement.

The paper is organised as follows. In Section 2, the general system description and the simulation model is introduced. In Section 3 the NDPO used to estimate the internal conditions of the system is presented. In Section 4, the NMPC strategy is stated and developed based on a simplified version of the PEMFC model introduced in Section 2. The simulation scenario and simulation results are presented in Section 5. Finally, Section 6 summarises the overall results of this paper and proposes some research lines for future work.

\section{Problem formulation}

\subsection{System description}

The system is presented in Figure 1 and it contains four main parts:

1. The PEMFC stack and load

2. The hydrogen delivery and recirculation auxiliaries

3. The air delivery and humidification auxiliaries

4. The refrigeration system

It is assumed that all the power is delivered by the fuel cell stack, henceforth, no additional power sources are considered. The hydrogen is stored in a high-pressure container. The cathode is fed with a compressor and the air is humidified before entering the stack.

Part of the power delivered by the PEMFC is used to feed the compressor and other auxiliaries. The total net electrical power delivered by the fuel cell system is expressed as

$$
P_{\text {net }}=P_{f c, e l e c}-P_{c m p}-P_{a u x}
$$

where $P_{f c, e l e c}$ is the gross electrical power generated by the fuel cell, $P_{c m p}$ the power consumed by the compressor and $P_{a u x}$ the total power consumption of the rest of auxiliary systems (hydrogen recirculation and refrigeration pumps and heat exchanger), which will be measured experimentally.

\subsection{PEMFC model}

The model presented in this section is the simulation model that emulates the fuel cell system in the present work. The control model is introduced in Section 4.1. The control model is a simplified version of the simulation model. This is done to improve the computational cost of the controller.

\subsubsection{Electrochemical model}

The PEMFC power can be modelled as the summation of the electrical and thermal power generation

$$
P_{f c}=P_{f c, e l e c}+P_{f c, t h}
$$


Table 1: Physical properties and constants

\begin{tabular}{|c|c|c|}
\hline Parameter & Description & Units \\
\hline$A$ & Area & $\mathrm{m}^{2}$ \\
\hline$c_{i}$ & Concentration of $i$-th gas & $\mathrm{mol} \mathrm{m}{ }^{-3}$ \\
\hline$D$ & Diffusion coefficient & $\mathrm{m}^{2} \mathrm{~s}^{-1}$ \\
\hline$E_{r}$ & Ideal fuel cell potential & $\mathrm{V}$ \\
\hline$F$ & Faraday constant & $\mathrm{C} \mathrm{mol}^{-} 1$ \\
\hline$I$ & Current & A \\
\hline$i$ & Current density & $\mathrm{A} \mathrm{m}^{-2}$ \\
\hline$i_{0}$ & Exchange current density & $\mathrm{A} \mathrm{m}^{-2}$ \\
\hline$K$ & Pressure drop coefficient & $\mathrm{m}^{2} \mathrm{~s}^{-1} \mathrm{~Pa}^{-1}$ \\
\hline$L$ & Fuel cell length & $\mathrm{m}$ \\
\hline$M$ & Molar mass & $\mathrm{g} \mathrm{mol}-1$ \\
\hline$\dot{n}_{i, i n}$ & Inlet molar flux of $i$-th gas & $\mathrm{mol} \mathrm{m}{ }^{-2} \mathrm{~s}^{-1}$ \\
\hline$\dot{n}_{i}$ & $\mathrm{y}$-direction flux of $i$-th gas & $\mathrm{mol} \mathrm{m}{ }^{-2} \mathrm{~s}^{-1}$ \\
\hline$n$ & Discretisation volumes & - \\
\hline$n_{c}$ & Number of cells in the stack & - \\
\hline$P$ & Power & $\mathrm{W}$ \\
\hline$p$ & Pressure & $\mathrm{Pa}$ \\
\hline$R$ & Gas constant & $\mathrm{J} \mathrm{mol}^{-1} \mathrm{~K}^{-1}$ \\
\hline$R_{o h m}$ & Membrane resistance & $\Omega$ \\
\hline$S$ & Source term & $\mathrm{kg} \mathrm{m}^{-3} \mathrm{~s}^{-1}$ \\
\hline$T$ & Temperature & K \\
\hline$V$ & Voltage & $\mathrm{V}$ \\
\hline$v$ & Flow velocity & $\mathrm{m} \mathrm{s}^{-1}$ \\
\hline$\alpha$ & Charge transfer coefficient & - \\
\hline$\Delta G_{0}$ & Gibbs activation energy & $\mathrm{J} \mathrm{mol}^{-1}$ \\
\hline$\Delta H$ & Hydrogen higher heating value & $\mathrm{J} \mathrm{mol}^{-1}$ \\
\hline$\delta$ & y-axis thickness & $\mathrm{m}$ \\
\hline$\varepsilon$ & Specific porosity & - \\
\hline$\eta$ & Efficiency & - \\
\hline$\omega$ & Angular speed & $\operatorname{rad~s}^{-1}$ \\
\hline$\rho$ & Water density & $\mathrm{kg} \mathrm{m}^{-3}$ \\
\hline
\end{tabular}




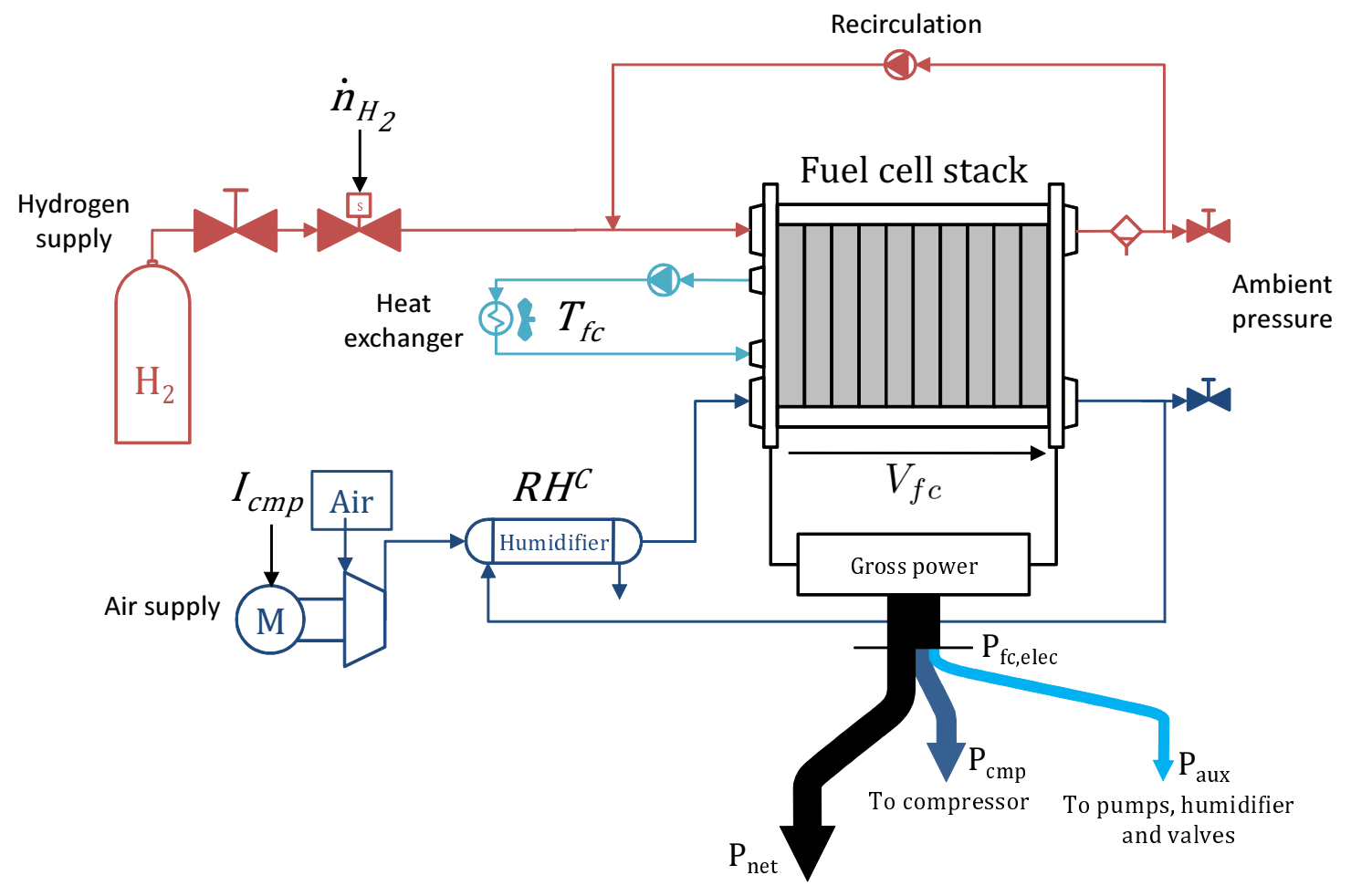

Figure 1: Diagram of the PEMFC-based power system 


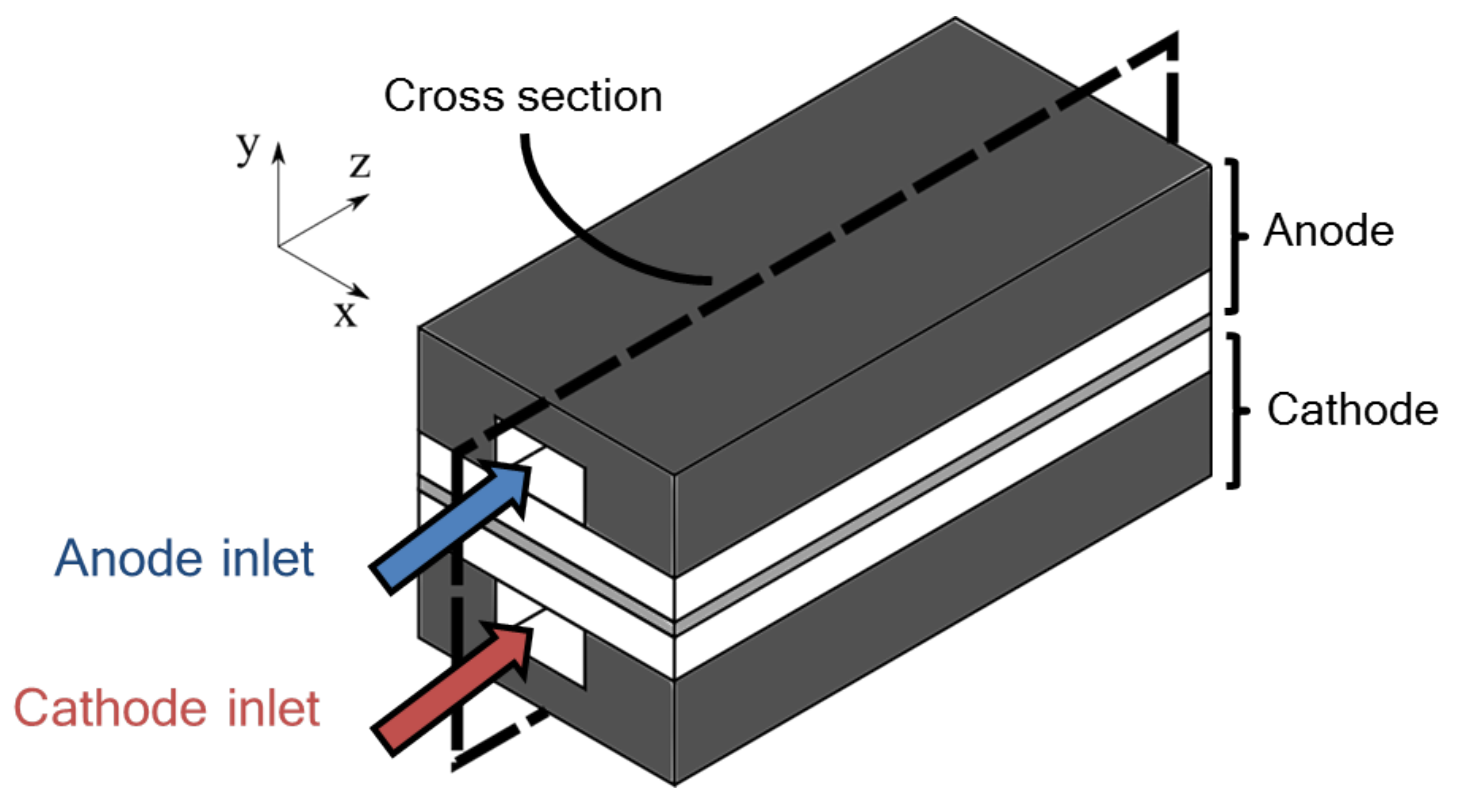

Figure 2: PEMFC single-channel representation

The fuel cell electrical power is expressed as

$$
P_{f c, e l e c}=V_{f c} I_{f c},
$$

being $V_{f c}$ the total fuel cell stack voltage and $I_{f c}$ the total current delivered by the PEMFC. Considering that the number of cells in the PEMFC stack is denoted by $n_{c}$,

$$
V_{f c}=V_{f c, \text { cell }} n_{c},
$$

where $V_{f c, \text { cell }}$ the individual cell voltage.

Then, using Eq. (4), Eq. (3) can be expressed as

$$
P_{f c, e l e c}=V_{f c, \text { cell }} I_{f c} n_{c} .
$$

The fuel cell current $I_{f c}$ is computed from the current density and is an input of the electrochemical model that can be written as

$$
i=\frac{I_{f c}}{A_{g e o}},
$$

being $A_{\text {geo }}$ the total surface area of the catalyst. This area is assumed to be the same for all the cells in the stack.

The single-cell voltage is modelled with the Butler-Volmer equation [28]

$$
V_{f c, \text { cell }}=E_{r}-\frac{R T}{\alpha 2 F}\left[\ln \left(\frac{i}{i_{0}}\right)-\ln \left(\frac{p_{O_{2}}}{p_{O_{2}}^{r e f}}\right)\right]-i R_{o h m},
$$


where $E_{r}$ is the ideal potential voltage of the fuel cell and $R_{o h m}$ is the internal resistance of the membrane that depends on the water content (see Section 2.2.4). Considering that the exchange current density at the anode is orders of magnitude larger tnan the one at the cathode [28], in this paper only the cathode activation and concentration losses are considered in Eq. (7). Henceforth, $\alpha$ is the cathode charge transfer coefficient and $p_{\mathrm{O}_{2}}$ and $p_{\mathrm{O}_{2}}^{r e f}$ are the oxygen pressure and oxygen pressure reference within the cathode catalyst layer (CCL) respectively.

In the simulation model, the exchange current density at the cathode $i_{0}$ includes the model developed in [27], which describes the electrochemical reaction kinetics taking into account the Electrochemically active Surface Area (ECSA) in the CCL $\left(\mathrm{ECSA}_{C C L}\right)$ :

$$
i_{0}=i_{0}^{r e f} \frac{E C S A_{C C L}}{A_{g e o}}\left(\frac{p_{O_{2}}}{p_{O_{2}}^{r e f}}\right)^{0.5} e^{\left[-\frac{\Delta G^{*}}{R T}\left(1-\frac{T}{T^{r e f}}\right)\right]},
$$

being $i_{0}^{r e f}$ the intrinsic catalytic Pt activity at normal conditions $T^{r e f}$ and $P_{\mathrm{O}_{2}}^{r e f}$ and $\Delta G^{*}$ the Gibbs activation energy for the oxygen reduction reaction.

While $A_{g e o}$ is the geometric surface area of the catalyst, the $\mathrm{ECSA}_{C C L}$ represents the active $\mathrm{Pt}$ area in the CCL. The $\mathrm{ECSA}_{C C L}$ is a function of the ratio of liquid volume to the total volume of void space in the porous CCL structure. This ratio is denoted by $s_{C C L}$. As explained in Section 2.2.3, $s_{C C L}$ depends on the formation of liquid water in the CCL and the two-phase water flow model. The computation of $s_{C C L}$ for a single pore size structural model is described in detail in [27].

\subsubsection{Gas flow model}

The gas species flow dynamics are described by mass balance equations along the PEMFC gas channels (see Figure 2)

$$
\begin{aligned}
\frac{\partial c_{i}}{\partial t} & =\frac{\partial}{\partial z}\left(v c_{i}\right)-\frac{\dot{n}_{i}}{\delta}+S_{i}^{g}, \\
v & =-K \frac{\partial p}{\partial z} \\
p & =R T \sum_{i} c_{i},
\end{aligned}
$$

where subscript $i$ stands for the gaseous species, namely $i=H_{2}$ for the hygrogen, $i=O_{2}$ for the oxygen, $i=\mathrm{N}_{2}$ for the nitrogen and $i=\mathrm{H}_{2} \mathrm{O}$ for the vapour water. The reaction and water molar transports from the membrane electrode assembly (MEA) are modelled in $\dot{n}_{i}$ and they are defined as perpendicular to the gas channels in the y-direction [3]. The $y$-direction depth of the anode and cathode gas channels is represented by $\delta$. The source term $S_{i}^{g}$ is only valid when $i=\mathrm{H}_{2} \mathrm{O}$ at the cathode side of the PEMFC $\left(S_{i}^{g}=0\right.$ for any other gaseous species and the water at the anode side); it represents the evaporation rate of the liquid water in the cathode side of the PEMFC (see Section 2.2.3).

The spatial derivative in Eq. (9) will be discretised to model the values of the gas concentrations in different sections along the gas channels. This will aid to implement control techniques that avoid global as well as local starvation in the PEMFC. Since it is not possible to measure the internal conditions of the fuel cell, a state observer is developed in Section 3 for the estimation of these internal variables. 


\subsubsection{Two-phase water model}

As introduced in Sections 2.2.1 and 2.2.2, the ratio of liquid water to vapour water in the CCL, denoted in this paper by $s_{C C L}$, affects the $\operatorname{ECSA}_{C C L}$ (which influences $i_{0}$ and $V_{f c, c e l l}$ ). Since only the cathode activation losses are considered in Eq. (8), the two-phase water model is implemented in the cathode side of the PEMFC (see Figure 4), disregarding the effect of liquid water at the anode side.

To be able to compute the ratio of liquid water in the CCL and GDL ( $s_{G D L}$ is necessary to obtain the boundary condition of $s_{C C L}$ ), first the general expression for the dynamics of $s$ is developed as introduced in $[27,29]$ :

$$
\frac{\partial s}{\partial t}=\frac{S_{H_{2} O}^{l}-\nabla \cdot\left(-D_{s} \vec{\nabla} s\right)}{\varepsilon \rho_{H_{2} O}^{l}},
$$

where $D_{s}$ denotes the liquid water diffusivity throughout the layer, $\varepsilon$ the specific porosity, $\rho_{\mathrm{H}_{2} \mathrm{O}}^{l}$ the liquid water density and $S_{\mathrm{H}_{2} \mathrm{O}}^{l}$ the liquid water source term (see Table 2) for the cathode side of the PEMFC. $\nabla \cdot \mathbf{F}$ and $\vec{\nabla} f$ denote the mathematical divergence and gradient respectively. Developing Eq. (10):

$$
\frac{\partial s}{\partial t}=\frac{S_{H_{2} O}^{l}+D_{s}\left(\frac{\partial^{2} s}{\partial x^{2}}+\frac{\partial^{2} s}{\partial y^{2}}+\frac{\partial^{2} s}{\partial z^{2}}\right)}{\varepsilon \rho_{H_{2} O}^{l} \mathrm{I}} .
$$

Notice that in Eq. (11) spatial derivatives in the $x, y$ and $z$-directions appear. In [27] only the $y$-direction discretisation was considered. In the present paper the approach will be implemented for the $y$ and $z$-directions. Therefore, disregarding the $x$-direction discretisation, Eq. (11) is expressed as

$$
\frac{\partial s}{\partial t}=\frac{S_{H_{2} O}^{l}+D_{s}\left(\frac{\partial^{2} s}{\partial y^{2}}+\frac{\partial^{2} s}{\partial z^{2}}\right)}{\varepsilon \rho_{H_{2} O}^{l} \mathrm{~S}} .
$$

To compute $S_{\mathrm{H}_{2} \mathrm{O}}^{l}$, the water generation $\left(S_{\mathrm{H}_{2} \mathrm{O}}^{\text {gen }}\right)$ and water evaporation $\left(S_{\mathrm{H}_{2} \mathrm{O}}^{\text {evp }}\right)$ terms are needed. While liquid water evaporation happens at the CCL and GDL, $S_{\mathrm{H}_{2} \mathrm{O}}^{g e n}$ only exists in the CCL. These source terms are modelled as follows:

$$
\begin{aligned}
S_{H_{2} \mathrm{O}}^{g e n} & =\gamma^{P t} \frac{i}{2 F} M_{\mathrm{H}_{2} \mathrm{O}}, \\
S_{\mathrm{H}_{2} \mathrm{O}}^{\text {evap }} & =K^{\text {evap }} s \frac{M_{\mathrm{H}_{2} \mathrm{O}}}{R T}\left(p^{\text {sat }}-p^{v}\right) \text { if } p^{v}<p^{\text {sat }},
\end{aligned}
$$

being $\gamma^{P t}$ the ECSA per unit of CCL volume [27] and $K^{\text {evap }}$ the water evaporation rate constant. An overview of the source terms is presented in Table 2. The evaporation source term in Eq. (14) is a function of the vapour pressure $p^{v}$ and the saturated vapour pressure $p^{\text {sat }}$ at a given temperature $T$

$$
p^{s a t}(T)=6.1121 e^{\left(18.678-\frac{T}{234.5}\right)\left(\frac{T}{257.14+T}\right)},
$$

as proposed by [30].

Following a similar procedure as in Section 2.2.2, Eq. (12) spatial derivatives are discretised. In the case of Eq. 12, this discretisation follows the $y$ and $z$ directions and as shown in Figure 4, it is done in the cathode catalyst and diffusion layers. After the discretisation, the water ratio at all the points of the discretisation grid in the cathode $\left(s_{C C L}\right)$ and gas diffusion $\left(s_{G D L}\right)$ layers is obtained. 
Table 2: Source terms for the cathode two-phase water model

\begin{tabular}{lll}
\hline & GDL & CCL \\
\hline$\left(\mathrm{H}_{2} \mathrm{O}\right)^{v}$ & $S_{\mathrm{H}_{2} \mathrm{O}}^{g}=S_{\mathrm{H}_{2} \mathrm{O}}^{\text {evap }}$ & $S_{\mathrm{H}_{2} \mathrm{O}}^{g}=S_{\mathrm{H}_{2} \mathrm{O}}^{\text {evap }}$ \\
$\left(\mathrm{H}_{2} \mathrm{O}\right)^{l}$ & $S_{\mathrm{H}_{2} \mathrm{O}}^{l}=-S_{\mathrm{H}_{2} \mathrm{O}}^{\text {evap }}$ & $S_{\mathrm{H}_{2} \mathrm{O}}^{l}=-S_{\mathrm{H}_{2} \mathrm{O}}^{\text {eva }}+S_{\mathrm{H}_{2} \mathrm{O}}^{\text {gen }}$ \\
\hline
\end{tabular}

\subsubsection{Membrane model}

The membrane layer model includes a complete water transport model [31]. Moreover, the membrane water content $(\Lambda)$ of the PEMFC is included in the model as the relation between the number of water moles and the moles of polymer in the membrane. The water content is obtained from the water partial pressures at both sides of the membrane [31].

The amount of accumulated water in the membrane affects the total ohmic resistance $R_{o h m}$ in Eq. (7). In this paper, the membrane resistance is modelled as follows [32]:

$$
R_{o h m}=\frac{\delta^{M}}{\sigma^{M}},
$$

where $\delta^{M}$ is the membrane thickness and $\sigma^{M}$ is the membrane conductivity, which is a function of the water content $\Lambda$ and the fuel cell temperature $T$ [31].

The water transport mechanisms that are modelled in the membrane are the electro-osmotic drag (EOD) and the back diffusion (BD) of the vapour water.

\subsection{Air supply system model}

The air-fed compressor nonlinear dynamics is described by the following set of ordinary differential equations [33]:

$$
\begin{aligned}
& \dot{p}_{O_{2}}=d_{1}\left(p_{s m}-p_{O_{2}}-p_{N_{2}}-d_{2}\right)-\frac{d_{3} p_{O_{2}}}{d_{4} p_{O_{2}}+d_{5} p_{N_{2}}+d_{6}} d_{17} \sqrt{p_{O_{2}}+p_{N_{2}}+d_{2}-d_{1} 1}-d_{7} I_{f c} \\
& \dot{p}_{N_{2}}=d_{8}\left(p_{s m}-p_{O_{2}}-p_{N_{2}}-d_{2}\right)-\frac{d_{3} p_{N_{2}}}{d_{4} p_{O_{2}}+d_{5} p_{N_{2}}+d_{6}} d_{17} \sqrt{p_{O_{2}}+p_{N_{2}}+d_{2}-d_{1} 1} \\
& \dot{\omega}_{c m p}=-d_{9} \omega_{c m p}-d_{10}\left[\left(\frac{p_{O_{2}}}{d_{11}}\right)^{d_{12}}-1\right] \dot{n}_{O_{2}}+d_{13} I_{c m p}, \\
& \dot{p}_{s m}=d_{14}\left[1+d_{15}\left[\left(\frac{p_{s m}}{d_{11}}\right)^{d_{12}}-1\right]\right] \times\left[\dot{n}_{O_{2}}-d_{16}\left(p_{s m}-p_{O_{2}}-p_{N_{2}}-d_{2}\right)\right]
\end{aligned}
$$

being $p_{\mathrm{O}_{2}}$ and $p_{N_{2}}$ the oxygen and nitrogen partial pressures respectively, $\omega_{c m p}$ the compressor angular speed and $p_{s m}$ the cathode channel supply manifold pressure. For simplicity, the constants of the compressor model, denoted by $d_{i}$, are defined in Appendix A.

The power consumed by the compressor $\left(P_{c m p}\right)$ is

$$
P_{c m p}=\tau_{c m p} \omega_{c m p}
$$


being $\tau_{c m p}$ and $\omega_{c m p}$ the torque and angular speed of the motor that powers the compressor respectively. The motor torque is

$$
\tau_{c m p}=\eta_{c m p} k_{t} I_{c m p},
$$

and therefore, Eq. (18) can be expressed as

$$
P_{c m p}=\eta_{c m p} k_{t} I_{c m p} \omega_{c m p},
$$

where $\eta_{c m p}$ is the compressor efficiency and $k_{t}$ the motor constant.

\subsection{Auxiliaries characterisation}

As seen in Figure 1, apart from the compressor, there are other systems that consume part of the power delivered by the PEMFC. In particular, these auxiliary systems are the following:

- Refrigeration pump

- Hydrogen recirculation pump

- Humidifier

- Valves

- Heat exchanger fan

The power consumption of the auxiliaries is not as significant as the power consumed by the compressor. While the detailed modelling of the aforementioned auxiliary systems is not needed, the corresponding current consumption characterisation $I_{a u x}$ will aid to complete the simulation model and obtain realistic results. Since the auxiliaries will be powered by the fuel cell, the total auxiliary consumed power can be expressed as

$$
P_{a u x}=V_{f c} I_{a u x},
$$

being $I_{a u x}$ the current consumed by all the auxiliaries.

To obtain this characterisation, a series of experiments have been done in a Bahia experiment bench [34]. This platform is equipped with sensors that can measure the power consumption of the auxiliary systems. In Figure 3 the required auxiliaries current density (without the compressor) as a function of the demanded NEDC load current density [35] is shown.

In Section 5, the auxiliaries, as well as the compressor current consumption, will be considered when calculating the net current delivered by the PEMFC system.

\subsection{Spatial discretisation}

As mentioned previously, the spatial derivatives present in Eqs. (9) and (12) are discretised using a forward-backward discretisation procedure [10]. While the discretisation of the partial differential equations allows to make use of lumped systems theory which takes advantage of the known boundary conditions, it introduces a high computational effort.

For the gas channels gas flow model, a new subscript $j$ is added to the state variables in Eq. (9). This subscript refers to the finite-element discretisation along the $z$-direction (e.g., $c_{H_{2}, 3}$ is the hydrogen concentration value at the third volume of the spatial discretisation). Ideally a fine discretisation would be the best. Nevertheless, the computational effort when increasing the 


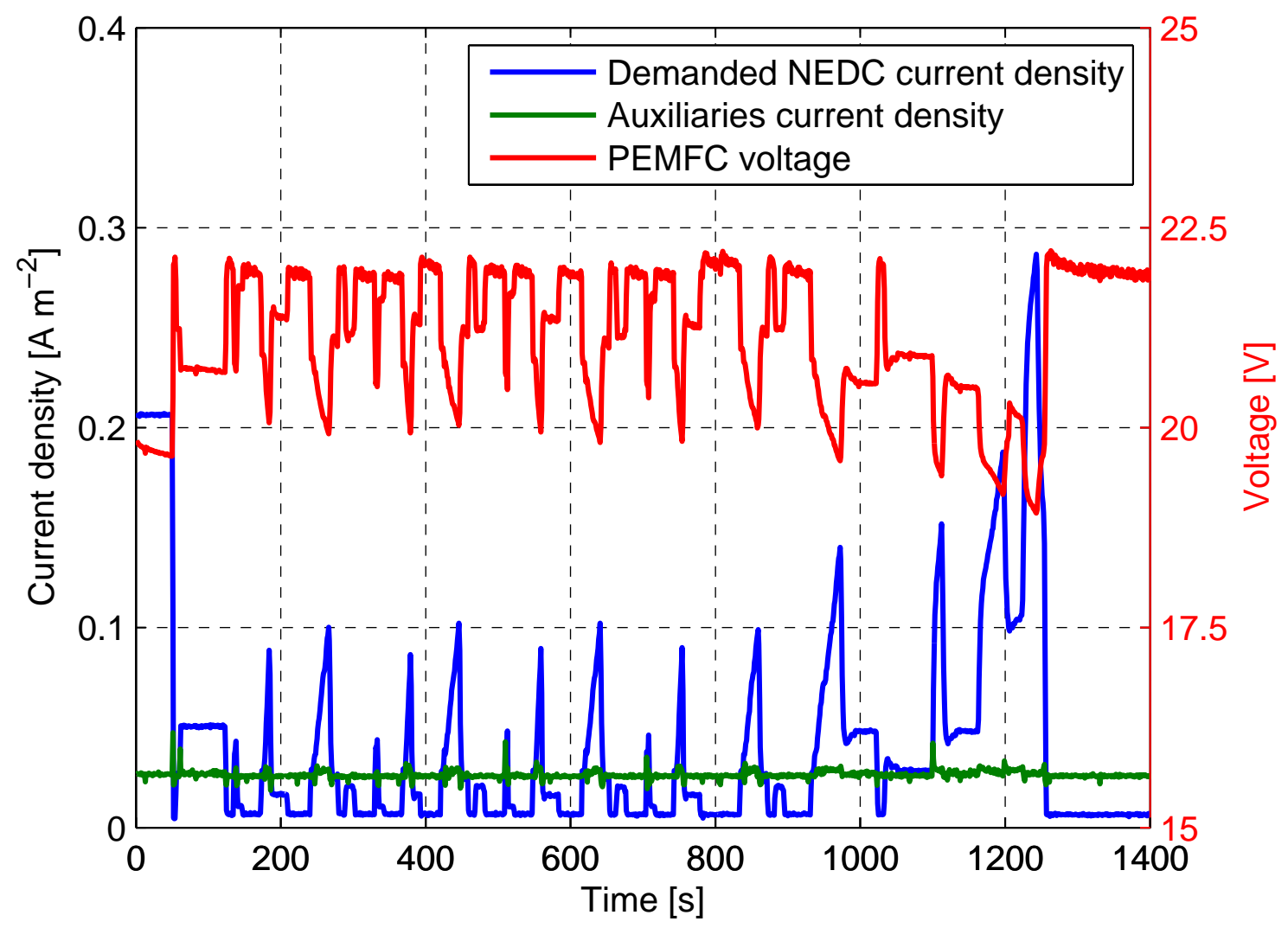

Figure 3: Auxiliaries current demand experiment 
number of discretisation volumes leads to determine the suitable trade-off between discretisation detail and computational burden. For this paper, the number of discretised volumes for the gas flow model is 5 .

The discretisation of the two-phase water model is done in the $y$ and $z$-directions. However, this model is not implemented in the prediction model of the NMPC, it is only considered in the simulation model. The discretisation volumes for the two-phase water model in Eq. (12) are 10 in the $y$-direction and 5 (same as in the gas flow model discretisation) in the $z$-direction.

The detailed discretised model along with the water transport mechanisms in the PEMFC are presented in Figure 4. A $z$-direction discretisation affecting all the PEMFC layers with a discretisation step of $\Delta z$ is performed as showed at the upper part of the figure. The $y$-direction discretisation of the cathode is highlighted with a red box at the bottom of Figure 4. Since the liquid water model presented in Section 2.2.3 is only implemented at the cathode side, there is no $y$-direction discretisation at the anode.

\section{Nonlinear state observer}

\subsection{Observation in PEMFC systems}

As previously explained, the enclosed construction of PEMFCs makes it impossible to perform certain measurements. Henceforth, there is no information about the values of all the states. However, reactant local starvation (low oxygen and hydrogen concentration values) can appear and can cause severe degradation in the system. Moreover, water content in the membrane greatly affects $V_{f c, c e l l}$. For this reason, a state observer is implemented using the measurement of some PEMFC outputs. In this paper a nonlinear distributed parameters observer (NDPO) $[9,10]$ is implemented. The NDPO approach will allow to consider the internal distribution of the reactants concentrations and the water content when designing the control strategy in Section 4. As mentioned in Section 2.2.4, the membrane water content $\Lambda$ is obtained from the water concentrations at the membrane boundaries.

Specifically, the knowledge of these internal concentration values will allow to design control laws that consider the problem of global as well as local starvation in the catalyst layers. The designed controller will decide which is the best action to improve the efficiency of the system while preventing the depletion of the reactants in the catalyst layers and therefore, enhancing the durability of the PEMFC.

\subsection{Observation model}

To estimate the internal gas concentration values in the PEMFC anode and cathode gas channels through a NDPO strategy, an observation model based on the gas flow model in Section 2.2.2 is developed. The discretisation of Eq. (9) over $n$ finite volumes $[9,10]$ along the gas channels direction for each one of the $i$-th gas species is:

$$
\dot{c}_{i, j}= \begin{cases}\frac{u_{i}}{\Delta z}-\zeta_{1} \Psi(j)-\frac{\dot{n}_{i, j}}{\delta}, & \text { if } j=1, \\ \zeta_{1} \Psi(j-1)+\Gamma(j+1)-\frac{\dot{n}_{i, j}}{\delta}, & \text { if } j=n, \\ \zeta_{1}(\Psi(j-1)-\Psi(j))-\frac{\dot{n}_{i, j}}{\delta}, & \text { else }\end{cases}
$$




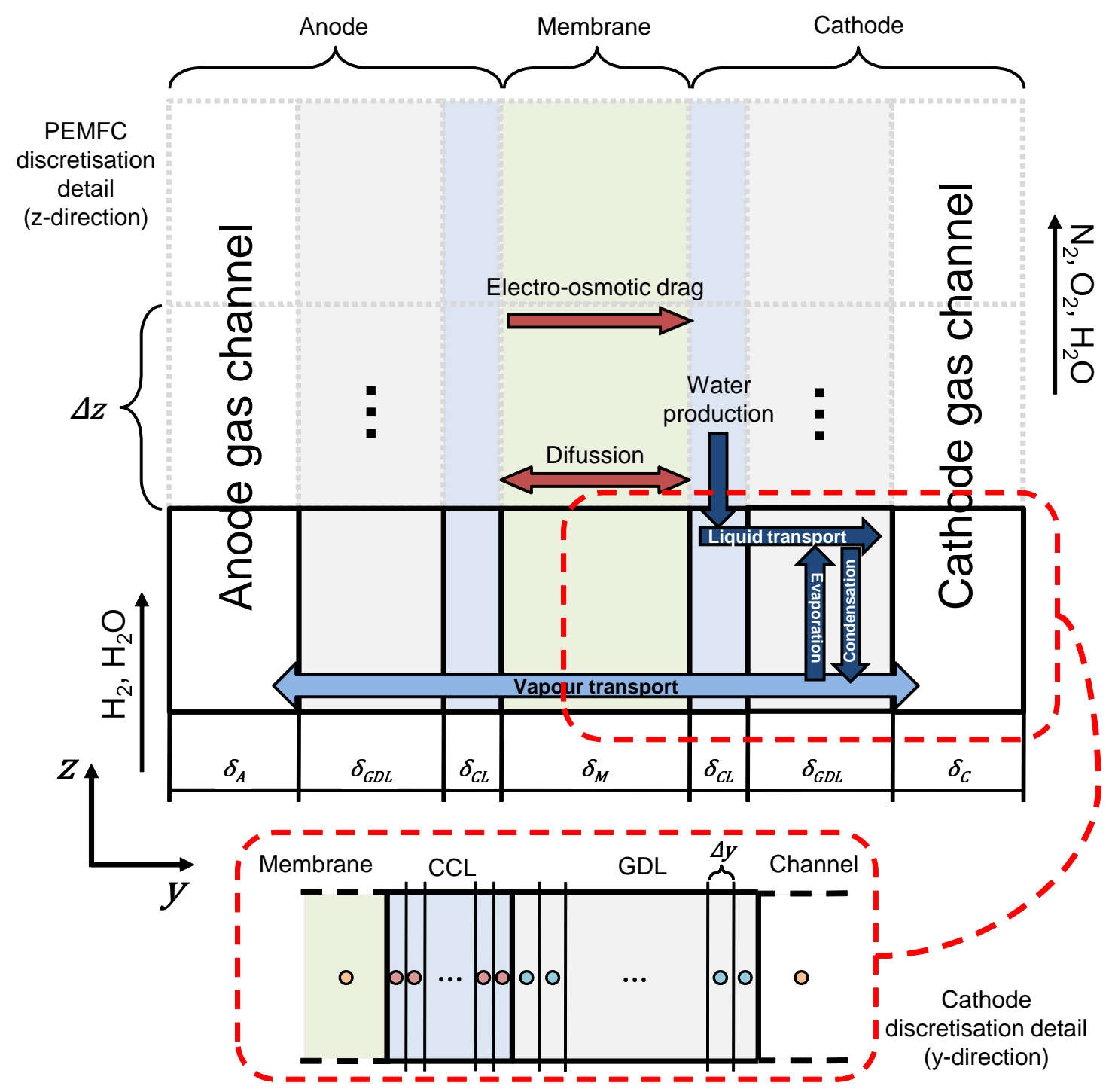

Figure 4: $z$ and $y$-direction discretisation detail and related two-phase water transport processes 
with

$$
\begin{aligned}
\Psi(j) & =c_{i, j}\left(\sum_{i} c_{i, j}-\sum_{i} c_{i, j+1}\right), \\
\Psi(j-1) & =c_{i, j-1}\left(\sum_{i} c_{i, j-1}-\sum_{i} c_{i, j}\right), \\
\Gamma(j+1) & =c_{i, j}\left(\zeta_{1} \sum_{i} c_{i, j}+\zeta_{2}\right),
\end{aligned}
$$

being $j \triangleq[1, \ldots, n], \zeta_{1}=K R T / \Delta z^{2}$ and $\zeta_{2}=K p^{a m b} / \Delta z^{2}$.

Eq. (22) has to be expressed in block controllable structure in order to apply the observation strategy $[10,36]$ proposed in Section 3.3:

$$
\left.\begin{array}{rl}
\dot{\hat{c}}_{i, 1} & =F_{i, 1}+B_{i, 1} u_{i, o b s, 1}+\hat{G}_{i, 1}, \\
& \vdots \\
\dot{\hat{c}}_{i, n-1} & =F_{i, n-1}+B_{i, n-1} \hat{c}_{i, n-2}+\hat{G}_{i n-1}, \\
\dot{\hat{c}}_{i, n} & =F_{i, n}+B_{i, n} \hat{c}_{i, n-1}+\hat{G}_{i, n},
\end{array}\right\}
$$

where $u_{i, o b s, 1}$ is the observation corrective action at the first volume (see Section 3.3), $\hat{c}_{i} \in \mathbb{R}^{5 \times n}$ is the observed state vector for all $i$-th gas species and $F_{i}:\{n\} \times\{1\} \in \mathbb{R}^{n}$ is the vector that includes the nonlinear dynamics that depend on the $j$ and $(j+1)$-discretisation volumes and the input molar flux for the $i$-th gas. Following the notation introduced in Eq. (22):

$$
F_{i}=\left[\begin{array}{c}
\frac{u_{i}}{\Delta z}-\zeta_{1} \hat{c}_{i, 1}\left(\sum_{i} \hat{c}_{i, 1}-\sum_{i} \hat{c}_{i, 2}\right) \\
-\zeta_{1} \hat{c}_{i, 2}\left(\sum_{i} \hat{c}_{i, 2}-\sum_{i} \hat{c}_{i, 3}\right) \\
\vdots \\
\hat{c}_{i, n}\left(\zeta_{1} \sum_{i} \hat{c}_{i, n}+\zeta_{2}\right)
\end{array}\right] .
$$

$B_{i}:\{n\} \times\{1\} \in \mathbb{R}^{n}$ is the vector that includes the nonlinear dynamics that depend on the $(j-1)$ discretisation volume

$$
B_{i}=\left[\begin{array}{c}
1 \\
\zeta_{1}\left(\sum_{i} \hat{c}_{i, 1}-\sum_{i} \hat{c}_{i, 2}\right) \\
\vdots \\
\zeta_{1}\left(\sum_{i} \hat{c}_{i, n-1}-\sum_{i} \hat{c}_{i, n}\right)
\end{array}\right]
$$

And finally, $\hat{G}_{i}:\{n\} \times\{1\} \in \mathbb{R}^{n}$ is the vector of the estimates for the reaction terms $\dot{n}_{i, j}$ in Eq. (22). These terms reflect the reactions perpendicular to the gas channels.

The output observation error for the $i$-th concentration value, namely $\bar{e}_{i, y}$, is

$$
\bar{e}_{i, y}=\hat{c}_{i, n}-c_{i, n} .
$$

The observation problem in this paper is to design a NDPO that drives Eq. (27) to zero in a finite amount of time and returns the full observed state vector for the $i$-th gas species at each discretisation volume. If Eq. (27) goes to zero in a finite amount of time, the full state observation error is also guaranteed to be approximately zero [10].

\subsection{NDPO back-stepping algorithm}

The proposed NDPO follows an $r$-step algorithm that depends on the number of discretisation volumes $(n)$, hence $r \in[1,2, \ldots, n]$. The back-stepping algorithm [10] to estimate the concentration 
values in Eq. (24) is:

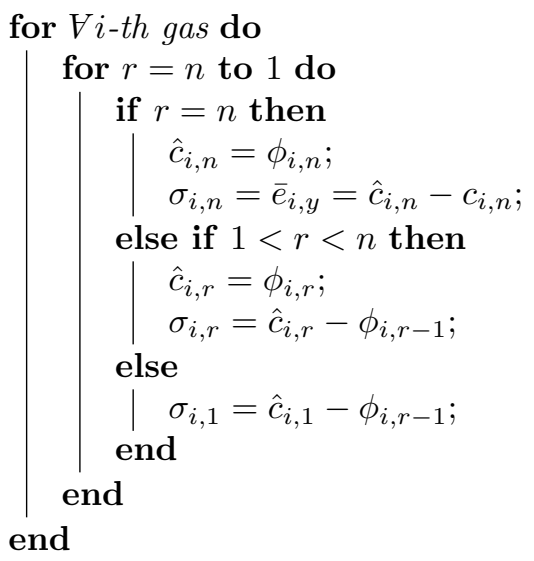

Algorithm 1: Back-stepping algorithm

The quasi-continuous term at each $r$ step $\left(\phi_{i, r}\right)$ in Algorithm 1 is defined as follows:

$$
\phi_{i, r}=B_{i, r}^{-1}\left\{-F_{i, r}+u_{i, o b s, r}\right\},
$$

having defined $F_{i}$ and $B_{i}$ in Eqs. (25) and (26) respectively.

The $r$-sliding homogeneous corrective action $u_{i, o b s, r}$ in Eq. (28) has to be designed for $r \leq n$

$$
u_{i, o b s, r}=-\alpha_{i, r} \Phi\left(\sigma_{i, r}, \dot{\sigma}_{i, r}, \ldots, \sigma_{i, r}^{(r-1)}\right),
$$

where $\Phi$ is the sliding surface that depends on the sign of the derivative terms $\dot{\sigma}, \ldots, \sigma^{(r-1)}$. The computation of the derivative terms is made using robust differentiators with finite-time convergence [37].

\subsection{Measurements}

To estimate the internal conditions of the PEMFC, it is assumed that the output concentration of each $i$-th gas species $\left(c_{i, n}\right)$ is available, as depicted in Algorithm 1. The strategy of this paper is that $c_{i, n}$ is inferred using measurements that are considered to be available to implement the NDPO:

- Relative humidity at the end of both gas channels

- Pressures at the end of both gas channels

- Input molar fluxes for all the gas species

- Temperature in the PEMFC

- Total output current

The details of how to infer the concentration values at the end of the anode and cathode gas channels from the previous measurements are explained in [9, 10]. Since the estimates depend on indirect measurements, a slight estimation error appears, although it can be considered negligible [10]. 


\section{NMPC controller design}

\subsection{Control-oriented model}

The NMPC strategy, as presented in Figure 5, uses a prediction model with its associated constraints. The differences between the simulation model presented in Section 2 and the prediction model are covered by the intrinsic robustness of the NMPC.

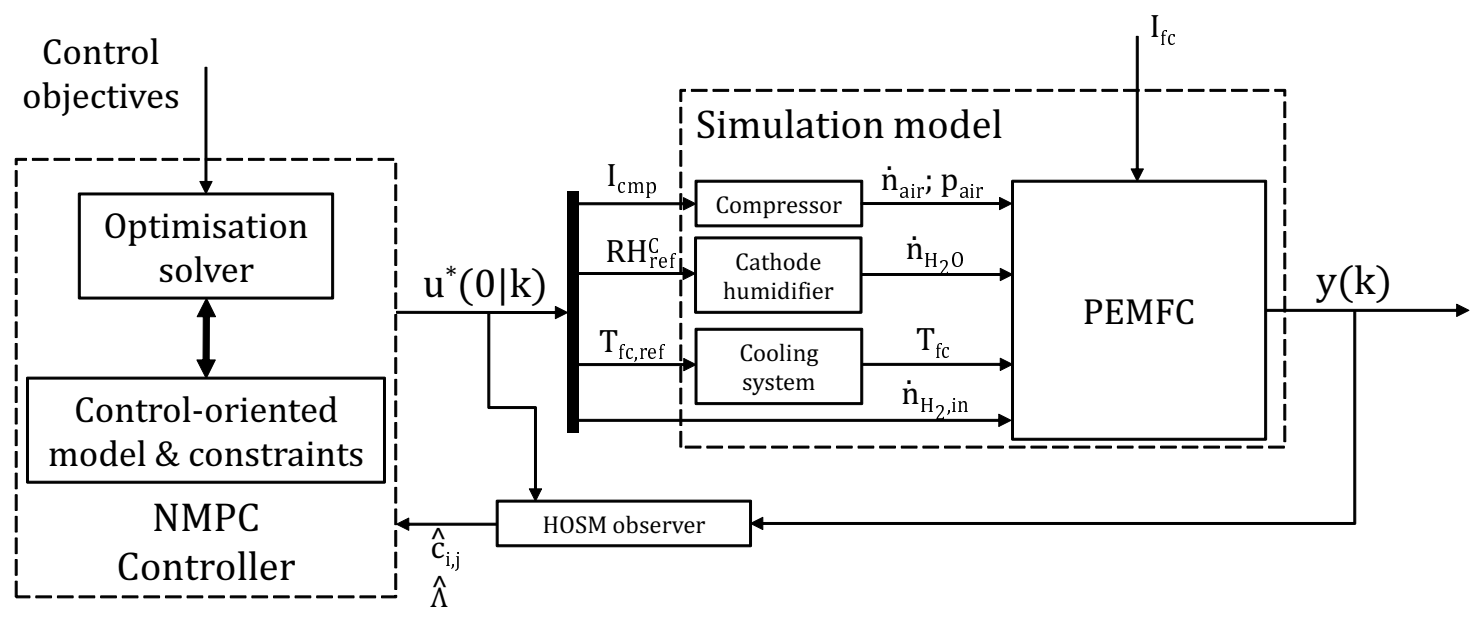

Figure 5: Closed-loop control scheme of the case study including input and output variables

$$
V_{f c, c e l l}(k)=E_{r}-\frac{R T_{f c}(k)}{\alpha 2 F} \times\left[\ln \left(\frac{i(k)}{i_{0}(k)}\right)-\ln \left(\frac{p_{O_{2}}(k)}{p_{O_{2}}^{r e f}}\right)\right]-i(k) R_{o h m}(k),
$$

where the discrete-time variable is denoted by $k \in \mathbb{Z}$.

As introduced in Section 2.5, the model is spatially discretised over $j$ volumes. Considering this, the discrete-time model for the gas channels of the PEMFC, derived from (9) is the following:

$$
c_{i, j}(k+1)=c_{i, j}(k)+\dot{c}_{i, j}(k) \Delta t,
$$

where the state variables are $c_{H_{2}, j}$ the hydrogen concentrations, $c_{O_{2}, j}$ the oxygen concentrations, $c_{N_{2}, j}$ the nitrogen concentrations and $c_{H_{2} O, j}$ the water concentrations (at both sides of the PEMFC), all along the $j$ discretised volumes of the gas channels. Moreover, as presented in Figure 5, the hydrogen input molar flow is a control input, denoted by $u_{H_{2}} \triangleq \dot{n}_{H_{2}, i n}$. The sampling time is defined by $\Delta t$ and for this paper it is $100 \mathrm{~ms}$.

From (17), the following discrete-time dynamic model of the compressor is obtained:

$$
\begin{aligned}
p_{O_{2}}(k+1) & =p_{O_{2}}(k)+\dot{p}_{O_{2}}(k) \Delta t, \\
p_{N_{2}}(k+1) & =p_{N_{2}}(k)+\dot{p}_{N_{2}}(k) \Delta t, \\
\omega_{c m p}(k+1) & =\omega_{c m p}(k)+\dot{\omega}_{c m p}(k) \Delta t, \\
p_{s m}(k+1) & =p_{s m}(k)+\dot{p}_{s m}(k) \Delta t,
\end{aligned}
$$


with the compressor current as a control input denoted by $u_{I_{c m p}} \triangleq I_{c m p}$.

\subsection{Control objectives}

The fuel cell has to provide the current demanded by the load and the auxiliaries of the system with maximum efficiency. The efficiency of the PEMFC-based system is

$$
\eta=\frac{P_{n e t}}{P_{t o t}}
$$

The power consumption of the system can be expressed as a function of the total hydrogen used to feed the system $\left(H_{2, \text { used }}\right)$ and the hydrogen higher heating value (HHV)

$$
P_{\text {tot }}=H_{2, \text { used }} \Delta H
$$

with $\Delta H$ as the HHV. Taking into consideration Eqs. (3, 20, 21 and 34) the efficiency of the system can be expressed as follows:

$$
\eta=\frac{P_{f c, e l e c}-P_{c m p}-P_{a u x}}{H_{2, \text { used }} \Delta H} .
$$

Maximising the overall system efficiency (35) is the first control objective of the NMPC strategy presented in this paper.

Moreover, to avoid fuel and oxidant starvation (which could cause permanent damage in the fuel cell [21]), it is necessary to maintain certain boundary values of $\mathrm{H}_{2}$ and $\mathrm{O}_{2}$ concentrations along the gas channels, no matter what the demanded power profile is. Moreover, it is important to properly hydrate the membrane (but not too much to avoid liquid water formation) to guarantee the suitable performance of the system; from this necessity arises the need to maintain the water content of the membrane between certain boundaries.

As explained before, the control objectives proposed in this paper aim to maintain given operating conditions in the PEMFC in order to avoid the global and local reactants starvation and the correct humidification of the membrane. To implement these control objectives the internal conditions have to be known. Since it is not possible to install sensors inside a PEMFC, using the NDPO presented in Section 3, these internal conditions will be estimated and included in the NMPC cost function.

\subsection{System constraints}

There are two types of system constraints considered in this paper: input and state constraints.

As presented in Figure 5, the input variables that are set by the NMPC are the compressor current $I_{c m p}$, the system reference temperature $T_{r e f}$, the input hydrogen molar flow $\dot{n}_{H_{2}, \text { in }}$ and the reference cathode relative humidity $R H_{\text {ref }}^{C}$. The input constraints depend mainly on the equipment employed (compressor and refrigeration systems) and the hydrogen mass flow controller. Here they are set as

$$
\begin{aligned}
& 1.2 \leq u_{I_{c m p}} \leq 3.5 \mathrm{~A}, \\
& 70 \leq u_{T_{\text {ref }}} \leq 90{ }^{\circ} \mathrm{C} \text {, } \\
& 0 \leq u_{H_{2}, \text { in }} \leq 100 \mathrm{~mol} \mathrm{~m}^{-2} \mathrm{~s}^{-1} \text {, } \\
& 0 \leq u_{R H_{\text {ref }}^{C}} \leq 1 \text {. }
\end{aligned}
$$


The values of the state constraints are fixed taking into account nominal values from models reported in the literature [3, 24]. Moreover, for the lower bounds of the hydrogen and oxygen concentrations, a procedure to know the minimum required concentration in the channel to diffuse through the GDLs up to the CLs has been performed by means of simulation under high current conditions. These constraints are

$$
\begin{aligned}
26 & <c_{H_{2}, j} \leq 45 \mathrm{~mol} \mathrm{~m}^{-3}, \forall j, \\
6 & <c_{\mathrm{O}_{2}, j} \leq 25 \mathrm{~mol} \mathrm{~m}^{-3}, \forall j, \\
0 & <c_{\mathrm{H}_{2} \mathrm{O}, j} \leq 6 \mathrm{~mol} \mathrm{~m} \mathrm{~m}^{-3}, \forall j,
\end{aligned}
$$

where (37a) is related to the hydrogen concentrations, (37b) to the oxygen concentrations and (37c) to the water concentrations. The control algorithm is flexible to introduce updated values, which can be extracted from the information given by the manufacturer of the system. Since all the discretisation volumes are constrained to have positive concentration values, there will always be enough $\mathrm{H}_{2}$ in the anode and $\mathrm{O}_{2}$ in the cathode gas channels to avoid local (condition guaranteed in all $j$ discretisation volumes) and global starvation in the system. Constraint (37c) is related to the vapour water concentration along the anode and cathode gas channels, affecting the humidification of the membrane and its water content.

\subsection{Cost function}

Given the control objectives stated in Section 4.2, the NMPC cost function that has to be minimised is:

$$
J(k)=\left\|\frac{1}{\eta}\right\|_{W_{e}}+\|\Delta u\|_{W_{u}}^{2},
$$

where $\Delta u=\left[\Delta u_{I_{c m p}}, \Delta u_{T_{r e f}}, \Delta u_{H_{2}, i n}, \Delta u_{R H_{r e f}^{C}}\right]^{T}$ with the slew-rate $\Delta u_{i}(k) \triangleq u_{i}(k)-u_{i}(k-1)$. Furthermore, notation $\|\cdot\|_{W_{i}}^{2}$ indicates the quadratic weighted norm, where the weighting matrices are defined as $W_{e}=\gamma_{e} \mathbb{I}$ and $W_{u}=\operatorname{diag}\left(\gamma_{u_{1}}, \gamma_{u_{2}}, \gamma_{u_{3}}, \gamma_{u_{4}}\right)$, with $\gamma_{u_{i}} \in \mathbb{R}$ and $\mathbb{I}$ being an identity matrix of suitable dimensions. Matrices $W_{i}$ allow to prioritise each control objective within the cost function (38).

Apart from the efficiency maximisation, terms for the slew-rate minimisation of the manipulable inputs have been included in (38), avoiding abrupt changes in the control inputs that could damage the system devices and accelerate their degradation [21].

\subsection{NMPC algorithm design}

Adopting the disturbance rejection problem proposed in [23], the NMPC algorithm is implemented. Therefore, the design of the NMPC for the proposed case study in this paper is based on Problem 1.

Problem 1 (NMPC Design). Let $^{1}$

$$
\mathbf{u}(k) \triangleq\left(u(0 \mid k), \ldots, u\left(H_{p}-1 \mid k\right)\right)
$$

\footnotetext{
${ }^{1}$ Here, $f(k+i \mid k)$ denotes the prediction of the variable $f$ at time $k+i$ performed at $k$. For instance, $x(k+i \mid k)$ denotes the prediction of the system state, starting from its initial condition $x(0 \mid k)=x(k)$.
} 
be the sequence of control inputs over a fixed-time prediction horizon $H_{p}$. Hence, the NMPC design is based on the solution of the finite-time open-loop optimization problem (FTOOP) depending also on the initial condition $x(0 \mid k) \triangleq x_{0}$

$$
\min _{\mathbf{u}(k) \in \mathbb{R}^{m H_{p}}} J\left(x_{0}, \mathbf{u}(k)\right),
$$

subject to

- system model in (30, 31 and 32) over $H_{p}$,

- input constraints in (36) over $H_{p}$,

- state constraints in (37) over $H_{p}$,

where $J(\cdot): \mathbb{U}^{m \times H_{p}} \times \mathbb{R}^{H_{p}} \mapsto \mathbb{R}$ in (38) is the cost function, with $m=4$ and $H_{p}=H_{u}$, where $H_{u}$ denotes the control horizon. Assuming that the FTOOP (40) is feasible, there will be an optimal solution for the sequence of control inputs

$$
\mathbf{u}^{*}(k) \triangleq\left(u^{*}(0 \mid k), u^{*}(1 \mid k), \ldots, u^{*}\left(H_{p}-1 \mid k\right)\right)
$$

and then, according to the receding horizon philosophy, $u_{i}^{*}(0 \mid k)$ is applied to the system, while the process is repeated for the next time instant $k \in \mathbb{Z}$.

Note that Problem 1 is a minimisation procedure. In the cost function (38) the efficiency of the system has been inverted to guarantee its maximum value in the optimisation.

\section{Simulation results}

\subsection{Numerical implementation}

The mesh for the simulation model and the nonlinear observer consists of 5 elements equally distributed along the $z$-direction (the simulation model has an extra $y$-direction discretisation for the CCL that is not reproduced in the control/observation models). The observer is initialised with the initial observer state vector $\hat{\mathbf{x}}_{\mathbf{0}}=\mathbf{0} \in \mathbb{R}^{5 \times n}$. Simulations have been carried out using fmincon function for MATLAB R2011a (32 bits), running in a PC Intel Core i7-3770 at $3.40 \mathrm{GHz}$ with 8 GB of RAM.

\subsection{Simulation scenario}

For the case study analysed in this paper, the stack is composed of $n_{c}=6$ identical singlechannel PEMFCs with a surface area $A_{\text {geo }}=25 \mathrm{~cm}^{2}$ for each one of the cells. The initial conditions of the simulation are defined by a given operating point described in Table 3. Initially the PEMFC is working under low stoichiometric values both at the anode and cathode sides with $50 \%$ relative humidity in the inlet channels.

To test the dynamic performance of the developed control strategy, an NEDC current profile [35] is going to be applied to the system. The employed NEDC current density profile is the same one used for the auxiliaries current demand experiment shown in Figure 3. 
Table 3: Initial operating conditions for the simulation

\begin{tabular}{clc}
\hline Condition & Unit & Value \\
\hline Anode stoichiometry & - & 1.3 \\
Anode inlet humidity & $\%$ & 50 \\
Anode inlet pressure & bar & 1.25 \\
Cathode stoichiometry & - & 2.0 \\
Cathode inlet humidity & $\%$ & 50 \\
Cathode inlet pressure & bar & 1.23 \\
Fuel cell temperature & $\mathrm{K}$ & 353 \\
\hline
\end{tabular}

\subsection{Controller setup}

One of the main capabilities of the NMPC strategy presented in this paper is the vast reconfigurability of the controller via the tuning of its parameters, including prediction and control horizons, penalisation terms, norms, etc. Table 4 shows the controller setup parameters and the sampling and simulation times used for the simulation scenario presented in the previous section.

Table 4: NMPC setup parameters

\begin{tabular}{clc}
\hline Parameter & Description & Value \\
\hline$\gamma_{e}$ & Efficiency maximisation penalisation & 1 \\
$\gamma_{u_{1}}$ & $I_{c m p}$ slew-rate penalisation & 1 \\
$\gamma_{u_{2}}$ & $T_{r e f}$ slew-rate penalisation & 1 \\
$\gamma_{u_{3}}$ & $\dot{n}_{H_{2}, \text { in }}$ slew-rate penalisation & 1 \\
$\gamma_{u_{4}}$ & $R H_{r e f}^{C}$ slew-rate penalisation & 1 \\
$H_{p}$ & Prediction horizon & 2 \\
$H_{c}$ & Control horizon & 2 \\
$\Delta t$ & Sampling time & $100 \mathrm{~ms}$ \\
$T_{s i m}$ & Simulation time & $1400 \mathrm{~s}$ \\
\hline
\end{tabular}

\subsection{Results and discussion}

The temporal behaviour of the efficiency defined in Eq. (33) is represented in Figure 6. As it can be seen in the figure, the NMPC implementation is clearly superior compared with the constant stoichiometry operation of the system (see Table 3). The controller allows the fuel cell to operate at lower stoichiometries with higher efficiency while guaranteeing that the PEMFC does not endure local starvation scenarios. 


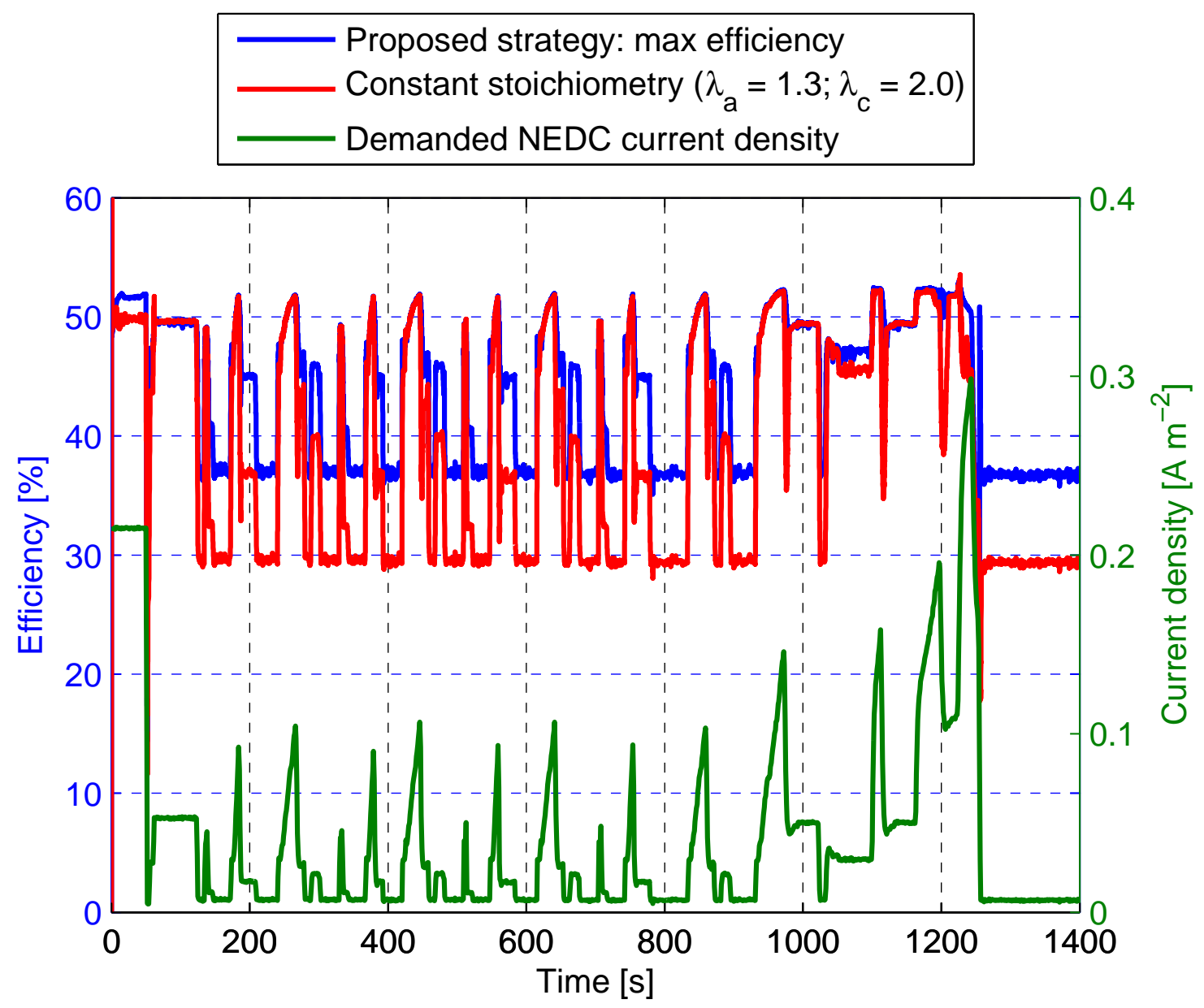

Figure 6: Efficiency of the system with the NMPC strategy and with constant operating conditions under a NEDC current demand 
The control actions are shown in Figure 7. These values are the optimal set for each simulation step as computed by the NMPC strategy. Moreover, as it can be seen in the figure, the values remain between the boundaries determined in Eq. (36).

a)

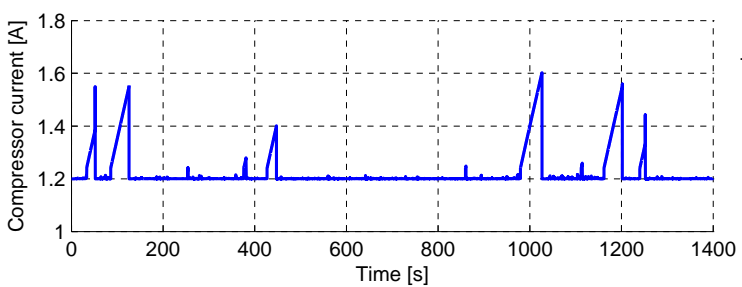

b)

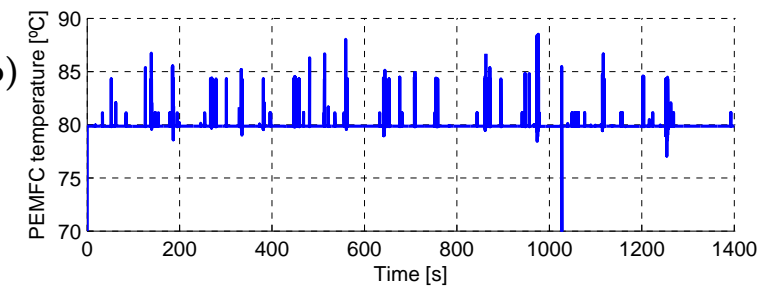

c)

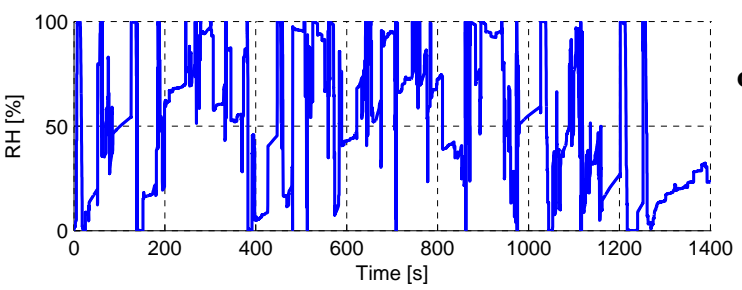

d)

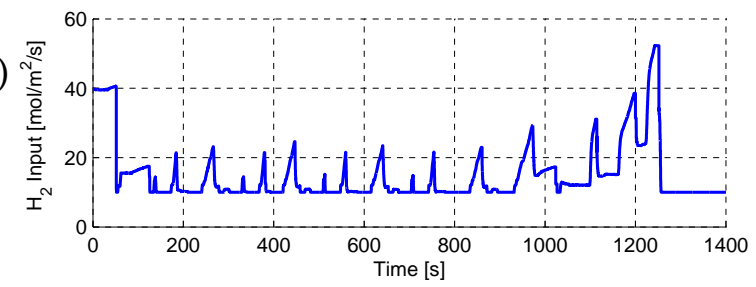

Figure 7: Compressor current (a), reference temperature (b), relative humidity (c) and $\mathrm{H}_{2}$ input molar flux (d) control actions

Regarding the degradation of the PEMFC, as mentioned in Section 1, three degradation categories can be distinguished: baseline, cycling and incident-induced degradation.

Since baseline degradation occurs always that a fuel cell is operated, in this paper the focus is set on the mitigation of cycling and incident-induced degradation. An important characteristic of the proposed NMPC strategy is the availability of setting the state constraints (Eqs. (36) and (37)). In this paper, the use of state constraints and a distributed parameters control model guarantees the avoidance of local starvation points at the anode and cathode sides of the PEMFC, thus preventing cycling as well as incident-induced degradation and not compromising the durability of the system. Regarding the cycling effects on degradation, it is possible to reduce them by means of a battery or super-capacitors, although this is out of the scope of this work. Slew-rates of the inputs, which limit the degree of variation that the system actuators can apply at each time step of the control horizon, also influence the system degradation. However, slew-rate values are difficult to set since some tradeoffs may appear. For example, limitation of the compressor current increase variation limits the reactants concentrations recovery but also avoids high air pressure transients at the cathode that can damage the membrane when the system is operating under highly cycling conditions, such as is the case of the NEDC. Moreover, in PEMFCs for mobile applications, there is a trade-off between the slew-rate and the drivability (the demanded power versus the delivered power relation) of the vehicle.

\subsection{Observer performance}

The NDPO feeds the NMPC controller with the estimated state vector as described in Section 3. This information is used by the controller to avoid local starvation in the fuel cell by guaranteeing that these values are between the desired restrictions (see Eq. (36)). Figure 8 shows the behaviour of the observed variables during the simulation time. While in Figure 8 only the observation of the 
concentrations in the middle point of the gas channels is presented, the observation is performed in all of the discretisation volumes. This is done to facilitate the reading of the results.

Figures $8 \mathrm{a}$ and $\mathrm{c}$, refer to the concentration estimation at the anode side. On the other hand, Figure $8 \mathrm{~b}$ and $\mathrm{d}$, refer to the cathode gas channel concentrations estimation. The estimation is robust during all the simulation as shown in Figure 8. Without the NDPO these internal variables would not be available to use in the control loop due to the impossibility of measuring inside the gas channels of the PEMFC.

In the case of Figures 8a and c, denoting the hydrogen and oxygen concentrations, the controller maintains these values between certain boundaries with the objective of avoiding local starvation. For the water concentrations in the anode and cathode sides of the PEMFC, depicted in Figures $8 \mathrm{~b}$ and $\mathrm{d}$, the controller guarantees that the humidification of the fuel cell is adequate without flooding the membrane.

a)

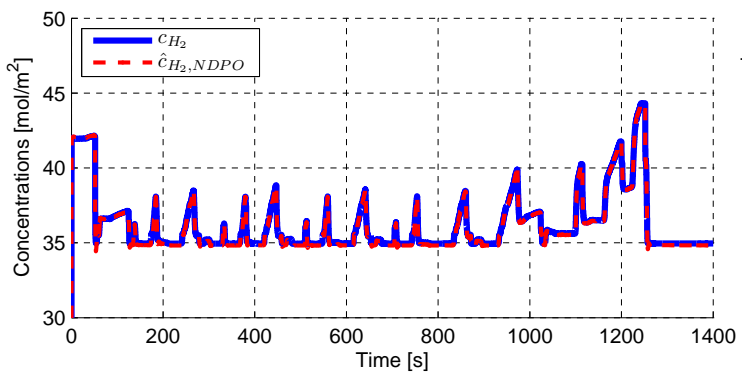

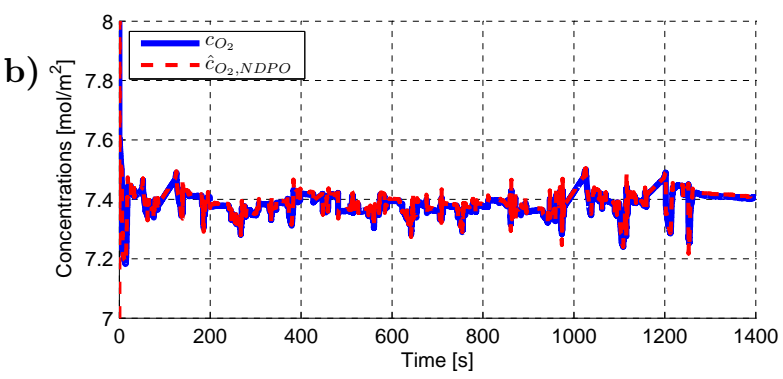

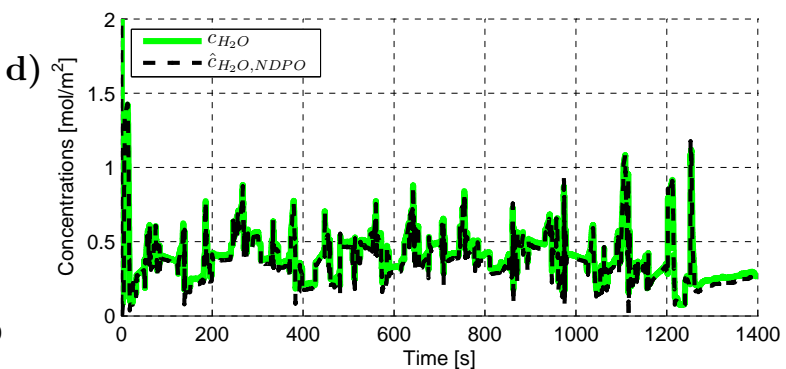

Figure 8: Behaviour of the NDPO versus the plant states in the middle discretisation volume of the anode (a and c) and the cathode ( $b$ and $d$ )

\section{Conclusions}

In this paper, a NMPC controller has been designed, developed and tested in simulation to operate a PEMFC-based power system. This approach allows to handle the nonlinear dynamics of the plant in order to track the demanded power profile (highly dynamic), to maximise efficiency and to avoid the degradation of the system by means of ensuring some minimum local concentrations of reactants and water in the catalyst layers. The performance of the controller has been evaluated, obtaining satisfactory results for a given simulation scenario.

The system representation includes the detailed modelling of the PEMFC (with different modelled layers), the compressor and the experimental mapping of the auxiliaries of the system. This 
allows to have reliable information about the behaviour of the system during the simulation of a NEDC current profile.

A total of five discretisation volumes have been considered to perform the control of the system. While it is possible to increase this value, a trade-off situation arises between the required level of detail in the controller and the mathematical complexity of the simulation model, which increases the computational burden.

The efficiency of the power system under the proposed closed-loop strategy (see Figure 6) is clearly improved. One of the main characteristics of the NMPC strategy is its high flexibility, due to different adjustable setup parameters combinations. A continuation of the approach proposed in this paper is to test different setup configurations and establish the fine tuning of the controller.

\section{Appendix A Air supply system}

A.1 Equations for the compressor model in Eq. (17)

$$
\begin{aligned}
d_{1} & =\frac{R T_{f c} K_{c a, i n}}{V_{c a} M_{O_{2}}} \frac{x_{O_{2}}}{\left(+\omega_{a t m}\right)} ; & d_{2} & =p_{\text {sat }} ; \\
d_{3} & =\frac{R T_{f c}}{V_{c a}} ; & d_{4} & =M_{O_{2}} ; \\
d_{5} & =M_{N_{2}} ; & d_{6} & =M_{v} p_{s a t} ; \\
d_{7} & =\frac{R T_{f c} n_{s t}}{V_{c a} 4 F} ; & d_{8} & =\frac{R T_{f c} k_{c a, i n}}{V_{c a} M_{N_{2}}} \frac{1-x_{O_{2}}}{\left(1+\omega_{a t m}\right)} ; \\
d_{9} & =\frac{f}{J_{c m p}} ; & d_{10} & =\frac{c_{p} T_{a m b}}{J_{c m p} \eta_{c m p}} ; \\
d_{11} & =p_{a t m} ; & d_{12} & =\frac{\gamma-1}{\gamma} ; \\
d_{13} & =\frac{\eta_{c m p} k_{t}}{J_{c m p}} ; & d_{1} 4 & =\frac{R T_{a t m}}{M_{a} V_{s m}} ; \\
d_{15} & =\frac{1}{\eta_{c m p}} ; & d_{16} & =k_{c a, i n} ; \\
d_{17} & =k_{c a, \text { out }} ; & d_{18} & =k_{c a, \text { in }} \frac{x_{O_{2}}}{\left(1+\omega_{a t m}\right)} ;
\end{aligned}
$$

\section{Acknowledgements}

This work has been partially funded by the Spanish national project MICAPEM (ref. DPI201569286-C3-2-R, MINECO/FEDER), the LABEX ACTION (ref. ANR-11-LABX-01-01) and the European project PUMA-MIND (ref. SPI-JTI-FCH 303419).

\section{References}

[1] M. Wöhr, K. Bolwin, W. Schnurnberger, M. Fischer, W. Neubrand, G. Eigenberger, Dynamic modelling and simulation of a polymer membrane fuel cell including mass transport limitation, International Journal of Hydrogen Energy 23 (3) (1998) 213-218.

[2] S. Um, C. Wang, K. Chen, Computational fluid dynamics modeling of proton exchange membrane fuel cells, Journal of the Electrochemical Society 147 (12) (2000) 4485-4493.

[3] M. Mangold, A. Bück, R. Hanke-Rauschenbach, Passivity based control of a distributed PEM fuel cell model, Journal of Process Control 20 (3) (2010) 292-313.

[4] M. Bavarian, M. Soroush, I. Kevrekidis, J. Benziger, Mathematical modeling, steady-state and dynamic behavior, and control of fuel cells: A review, Industrial \& Engineering Chemistry Research 49 (17) (2010) 7922-7950. 
[5] C. Bordons, A. Arce, A. Del Real, Constrained predictive control strategies for PEM fuel cells, in: American Control Conference, IEEE, 2006, pp. 6-pp.

[6] C. Kunusch, P. Puleston, M. Mayosky, A. Husar, Control-oriented modeling and experimental validation of a PEMFC generation system, IEEE Transactions on Energy Conversion 26 (3) (2011) 851-861.

[7] C. Damour, M. Benne, C. Lebreton, J. Deseure, B. Grondin-Perez, Real-time implementation of a neural model-based self-tuning PID strategy for oxygen stoichiometry control in PEM fuel cell, International Journal of Hydrogen Energy 39 (24) (2014) 12819-12825.

[8] C. Kunusch, A. Husar, P. Puleston, M. Mayosky, J. Moré, Linear identification and model adjustment of a PEM fuel cell stack, International Journal of Hydrogen Energy 33 (13) (2008) $3581-3587$.

[9] J. Luna, A. Husar, M. Serra, Nonlinear distributed parameter observer design for fuel cell systems, International Journal of Hydrogen Energy 40 (2015) 11322-11332.

[10] J. Luna, E. Usai, A. Husar, M. Serra, Nonlinear observation in fuel cell systems: A comparison between disturbance estimation and high-order sliding-mode techniques, International Journal of Hydrogen Energydoi:10.1016/j.ijhydene.2016.06.041.

[11] A. Pilloni, A. Pisano, E. Usai, Observer based air excess ratio control of a PEM fuel cell system via high order sliding mode, IEEE Transactions on Industrial Electronics.

[12] D. Friedman, R. Moore, PEM fuel cell system optimization, Proceedings Electrochemical Society 27 (1998) 407-423.

[13] M. Grujicic, K. Chittajallu, E. Law, J. Pukrushpan, Model-based control strategies in the dynamic interaction of air supply and fuel cell, Proceedings of the Institution of Mechanical Engineers 218 (7) (2004) 487-499.

[14] J.-W. Ahn, S.-Y. Choe, Coolant controls of a PEM fuel cell system, Journal of Power Sources 179 (1) (2008) 252-264.

[15] I. Matraji, S. Laghrouche, M. Wack, Pressure control in a PEM fuel cell via second order sliding mode, International Journal of Hydrogen Energy 37 (21) (2012) 16104-16116.

[16] Y. Shao, G. Yin, Y. Gao, Understanding and approaches for the durability issues of pt-based catalysts for PEM fuel cell, Journal of Power Sources 171 (2) (2007) 558-566.

[17] X. Yu, S. Ye, Recent advances in activity and durability enhancement of pt/c catalytic cathode in PEMFC: Part II: Degradation mechanism and durability enhancement of carbon supported platinum catalyst, Journal of Power Sources 172 (1) (2007) 145-154.

[18] J. Wang, G. Yin, Y. Shao, S. Zhang, Z. Wang, Y. Gao, Effect of carbon black support corrosion on the durability of pt/c catalyst, Journal of Power sources 171 (2) (2007) 331-339.

[19] S. Zhang, X. Yuan, H. Wang, W. Mérida, H. Zhu, J. Shen, S. Wu, J. Zhang, A review of accelerated stress tests of MEA durability in PEM fuel cells, International Journal of Hydrogen Energy 34 (1) (2009) 388-404. 
[20] Y. Li, T. Zhao, Understanding the performance degradation of anion-exchange membrane direct ethanol fuel cells, International Journal of Hydrogen Energy 37 (5) (2012) 4413-4421.

[21] N. Yousfi-Steiner, P. Moçotéguy, D. Candusso, D. Hissel, A review on polymer electrolyte membrane fuel cell catalyst degradation and starvation issues: Causes, consequences and diagnostic for mitigation, Journal of Power Sources 194 (1) (2009) 130-145.

[22] J. M. Maciejowski, Predictive control: with constraints, Pearson Education, 2002.

[23] L. Grüne, J. Pannek, Nonlinear model predictive control, Springer, 2011.

[24] J. Luna, C. Ocampo-Martinez, M. Serra, Nonlinear predictive control for the concentrations profile regulation under unknown reaction disturbances in a fuel cell anode gas channel, Journal of Power Sources 282 (2015) 129-139.

[25] C. Kunusch, P. Puleston, M. Mayosky, A. Dávila, Efficiency optimisation of an experimental PEM fuel cell system via super twisting control, in: 11th International Workshop on Variable Structure Systems (VSS), IEEE, 2010, pp. 319-324.

[26] Q. Li, W. Chen, Y. Wang, J. Jia, M. Han, Nonlinear robust control of proton exchange membrane fuel cell by state feedback exact linearization, Journal of Power Sources 194 (1) (2009) 338-348.

[27] S. Strahl, A. Husar, A. A. Franco, Electrode structure effects on the performance of opencathode proton exchange membrane fuel cells: A multiscale modeling approach, International Journal of Hydrogen Energy 39 (18) (2014) 9752-9767.

[28] F. Barbir, PEM fuel cells: theory and practice, Elsevier Academic Press, 2013.

[29] H. Wu, X. Li, P. Berg, On the modeling of water transport in polymer electrolyte membrane fuel cells, Electrochimica Acta 54 (27) (2009) 6913-6927.

[30] A. Buck, New equations for computing vapor pressure and enhancement factor, Journal of Applied Meteorology 20 (12) (1981) 1527-1532.

[31] W. Neubrand, Modellbildung und Simulation von Elektromembranverfahren, Logos-Verlag, 1999.

[32] J. T. Pukrushpan, H. Peng, A. G. Stefanopoulou, Simulation and analysis of transient fuel cell system performance based on a dynamic reactant flow model, in: ASME 2002 International Mechanical Engineering Congress and Exposition, American Society of Mechanical Engineers, 2002, pp. 637-648.

[33] S. Laghrouche, I. Matraji, F. S. Ahmed, S. Jemei, M. Wack, Load governor based on constrained extremum seeking for pem fuel cell oxygen starvation and compressor surge protection, International Journal of Hydrogen Energy 38 (33) (2013) 14314-14322.

[34] Bahia Bench V2.1-A1, http://www.areva.com/EN/operations-4576/ bahia-bench-overview.html. 
[35] M. Mayur, S. Strahl, A. Husar, W. G. Bessler, A multi-timescale modeling methodology for pemfc performance and durability in a virtual fuel cell car, International Journal of Hydrogen Energy 40 (46) (2015) 16466-16476.

[36] A. Estrada, L. Fridman, Quasi-continuous HOSM control for systems with unmatched perturbations, Automatica 46 (11) (2010) 1916-1919.

[37] A. Levant, Higher-order sliding modes, differentiation and output-feedback control, International Journal of Control 76 (9-10) (2003) 924-941. 